\title{
RELAÇÃO ENTRE FLUXOS DE CAIXA LIVRES E NÍVEIS DE GOVERNANÇA CORPORATIVA À LUZ DA TEORIA DA AGÊNCIA
}

\section{RELATIONSHIP BETWEEN FREE CASH FLOWS AND CORPORATE GOVERNANCE LEVELS IN THE LIGHT OF AGENCY THEORY}

\author{
GLAYSSON AGUILAR DE ARAÚJO \\ Universidade Federal de Minas Gerais. Endereço: Avenida \\ Antônio Carlos, 6.627 | Pampulha | 31270-901 | Belo \\ Horizonte/MG | Brasil. \\ (D) https://orcid.org/0000-0002-7774-2227 \\ glayssonaraujo@yahoo.com.br
}

\section{LARA ALVES CORREAA}

Universidade Federal de Minas Gerais. Endereço: Avenida Antônio Carlos, 6.627 | Pampulha | 31270-901 | Belo Horizonte/MG | Brasil.

(D) https://orcid.org/0000-0002-6495-3615

laradbh@hotmail.com

\section{VALÉRIA GAMA FULLY BRESSAN}

Universidade Federal de Minas Gerais. Endereço: Avenida Antônio Carlos, 6.627 | Pampulha | 31270-901 | Belo Horizonte/MG | Brasil.

(1) https://orcid.org/0000-0001-6340-9717

vfully@face.ufmg.br

\section{JOÃO ESTEVÃO BARBOSA NETO}

Universidade Federal de Minas Gerais. Endereço: Avenida Antônio Carlos, 6.627 | Pampulha | 31270-901 | Belo Horizonte/MG | Brasil.

(1) https://orcid.org/0000-0001-5197-2166

joaoestevaobarbosaneto@gmail.com

\section{BRUNA CAMARGOS AVELINO}

Universidade Federal de Minas Gerais. Endereço: Avenida Antônio Carlos, 6.627 | Pampulha | 31270-901 | Belo Horizonte/MG | Brasil.

(1) https://orcid.org/0000-0001-8958-8725

bcavelino@gmail.com

\section{RESUMO}

Esta pesquisa analisa a relação entre os Fluxos de Caixa Livres (FCLs) e os diferentes níveis de Governança Corporativa presentes no mercado acionário brasileiro. Para tanto, a amostra foi composta por 212 empresas brasileiras de capital aberto listadas na Brasil, Bolsa, Balcão [B]³, no período de 2010 a 2018. A metodologia consistiu na estimação de uma regressão para dados em painel, com a utilização do modelo para efeitos aleatórios, estimando-se por mínimos quadrados generalizados (generalized least square - GLS) e assumindo os ajustes para autocorrelação e erros padrão robustos para heterocedasticidade. Os resultados encontrados, para a amostra estudada, sugerem que os níveis de Governança Corporativa apresentam relação positiva com os FCLs. Em

Editado em português e inglês. Versão original em português.

Artigo apresentado no XX USP International Conference in Acconting, em São Paulo, de 29 a 31 de julho de 2020.

Recebido em 15/9/2021. Revisado em 8/11/2021. Aceito em 16/11/2021 pelos Prof. Dr. Sérgio Murilo Petri (Editor-Chefe) e Prof. Dr. Sandro Vieira Soares (Editor Adjunto). Publicado em 30/11/2021.

Copyright ( 2021 RCCC. Todos os direitos reservados. É permitida a citação de parte de artigos sem autorização prévia, desde que identificada a fonte. 
sinergia, quando comparadas ao nível Tradicional da $[\mathrm{B}]^{3}$, as empresas listadas nos níveis Novo Mercado e Nível 2 tendem a apresentar maiores valores de FCL. Acrescenta-se, ainda, que, quanto maior o tamanho das empresas e quanto maior o retorno sobre o patrimônio líquido, maiores tendem a ser os FCLs, assim como empresas em estágios de amadurecimento tendem a apresentar menores valores de FCLs. A relevância desta pesquisa pauta-se em analisar, em um mercado acionário sujeito a imperfeições, fatores que possam afetar decisões sobre o nível de manutenção de caixa das empresas, mais especificamente ao avaliar como os mecanismos de Governança Corporativa relacionam-se com a teoria dos FCLs, num contexto de potencial conflito de interesses.

Palavras-chave: Fluxos de Caixa Livres. Teoria da agência. Governança Corporativa.

\begin{abstract}
This research analyzes the relationship between free cash flows (FCFs) and the different levels of Corporate Governance present in the Brazilian stock market. To this end, the sample was composed of 212 Brazilian publicly traded companies listed on Brasil, Bolsa, Balcão [B] $]^{3}$, in the period from 2010 to 2018. The methodology consisted of estimating a regression for panel data, using the random effects model, estimating by generalized least square (GLS) and assuming adjustments for autocorrelation and robust standard errors for heteroscedasticity. The results found, for the sample studied, suggest that Corporate Governance levels are positively related to the FCFs. In synergy, when compared to the Traditional level of $[B]^{3}$, companies listed on the Novo Mercado and Level 2 levels tend to present higher FCF values. In addition, the larger the size of the companies and the higher their return on equity, the higher their FCFs tend to be, just as companies in stages of maturity tend to present lower FCF values. The relevance of this research is based on analyzing, in a stock market subject to imperfections, factors that may affect decisions about the level of cash maintenance of companies, more specifically by evaluating how Corporate Governance mechanisms relate to the theory of FCFs, in a context of potential conflict of interest.
\end{abstract}

Keywords: Free cash flows. Agency theory. Corporate Governance.

\title{
1 INTRODUÇÃO
}

No contexto do moderno ambiente empresarial, as corporações sofreram significativas mudanças, pois a estrutura societária das empresas, anteriormente concentrada, passou a ser pulverizada em diversos acionistas. E a gerência, exercida inicialmente pelos detentores do capital, passou a ser delegada a outros, que administram o capital investido pelos acionistas (Martin, Santos \& Dias Filho, 2004). Nesse cenário, destacam-se os fundamentos da Teoria da Agência, na qual Jensen e Meckling (1976, p. 308) conceituam "uma relação de agência como um contrato sob o qual uma ou mais pessoas (principal (is)) emprega uma outra pessoa (agente) para executar em seu nome um serviço que implique confiar o poder de decisão ao agente". Especificamente, o mercado acionário brasileiro apresenta como característica uma alta concentração acionária, sendo tal fato um elemento potencial de conflitos entres agentes (Machado \& Galdi, 2021).

Os gerentes corporativos são os agentes dos acionistas, o que pode ocasionar um relacionamento repleto de interesses conflitantes entre acionistas e gestores, sendo que estes últimos podem agir visando seus interesses pessoais para tomar vantagem na gestão das empresas. Sob essa ótica, um dos instrumentos que pode ser utilizado pelos gestores é o Fluxo de Caixa Livre (FCL), considerado como o conjunto dos fundos disponíveis, após o financiamento de todos os 
projetos com valor atual líquido positivo, atualizados ao custo de oportunidade do capital (Jensen, 1986).

Os agentes utilizam o FCL de forma divergente aos interesses dos acionistas como, por exemplo, para investir em projetos duvidosos, consumir gratificações desnecessárias e gerenciar resultados visando a autopromoção (Jensen, 1986). Não obstante, o FCL, se gerido de forma eficiente, pode ser visto como um importante fator contributivo para a persistência de bons resultados e, por isso, tem chamado a atenção de pesquisadores (Wang, 2010).

Ferreira e Vilela (2004) contextualizam as seguintes variáveis como determinantes do fluxo de caixa: i) conjunto de oportunidades de investimentos: espera-se que gerentes de empresas com poucas oportunidades de investimento retenham mais recursos para aplicar em projetos de crescimento, mesmo que o valor presente líquido desses projetos seja negativo, o que pode ocasionar a destruição de valor para o acionista; ii) alavancagem: empresas menos alavancadas estão menos expostas a monitoramento, o que permite maior discricionariedade na gestão; e iii) tamanho: há uma maior dispersão acionária em empresas de maior porte, o que permite que os gestores possuam maiores poderes implícitos.

Diante desse contexto, com o intuito de mitigar prejuízos decorrentes de conflitos de interesses entre o agente e o principal, foram criados os mecanismos de Governança Corporativa. Presume-se que, por intermédio da adoção de boas práticas de Governança Corporativa, os recursos dos acionistas sejam empregados de forma eficiente e eficaz na missão, com vistas a alcançar os objetivos e metas da organização, assim como maximizar os retornos esperados (Arruda, Madruga \& Freitas Junior, 2008).

Para Jensen (1986), as empresas com poucos mecanismos de Governança Corporativa investem seu excesso de caixa ineficientemente; alternativamente, propõe que o excesso de caixa simplesmente reduz as pressões sobre a administração para controlar custos, melhorar margens, monitorar de perto os funcionários e as operações e, geralmente, aumentar os lucros. Portanto, supõe-se que as empresas mal geridas dissipam o caixa mais rapidamente do que as empresas bem governadas, operando de maneira a reduzir os retornos contábeis da entidade.

Pesquisas (Dittmar \& Mahrt-Smith, 2007; McKnight \& Weir, 2009; Dylewski, 2010; Chi \& Lee, 2010 e Wang, 2010) avaliaram a capacidade dos mecanismos de Governança Corporativa na limitação dos problemas decorrentes de FCL, tendo como base a hipótese de Jensen (1986) de que a Governança Corporativa reduziria o FCL. Ou seja, empresas com mais altos níveis de Governança Corporativa apresentariam menores FCL. Jensen (1986) argumenta que a presença de FCL pode levar ao desperdício e à ineficiência gerenciais e, por isso, os detentores do capital implantam mecanismos de controle para garantir seus interesses.

Alguns desses estudos apresentaram resultados que corroboraram as hipóteses de Jensen (1986). Dittmar e Mahrt-Smith (2007), por exemplo, encontraram evidências da influência da Governança Corporativa sobre as sobras de caixas. Chi e Lee (2010) encontraram evidências de que a Governança Corporativa afeta o valor da empresa de maneira diferente, dependendo se a entidade possui FCL alto ou baixo. Já outros estudos contrariam as hipóteses propostas por Jensen (1986). Wang (2010) verificou que os custos de agência estão associados negativamente ao desempenho da empresa, enquanto o FCL está relacionado positivamente. McKnight e Weir (2009) elucidaram que as mudanças nas estruturas dos conselhos não têm impacto nos custos de agência e que ter um comitê de nomeação de diretores que, por sua vez, utiliza o critério de meritocracia, aumenta, ao invés de reduzir, os custos de agência. Por sua vez, Dylewski (2010) encontrou resultados que apontam que empresas com nível de Governança Corporativa mais elevado possuem maiores níveis de caixa do que as demais.

Trabalhos como os de Li e Song (2018), Gupta, Mahakud e Verma (2021) e Sprenger e Lazarevab (2021) deram destaque à sensibilidade investimento-fluxo de caixa. Li e Song (2018) testaram, especificamente, os efeitos da proteção anti-takeover na sensibilidade do investimento 
ao fluxo de caixa e verificaram que tal sensibilidade é maior quando os gestores estão isolados de takeovers/aquisições. Ainda, verificaram que os efeitos da aprovação de leis anti-takeover sobre a sensibilidade do investimento ao fluxo de caixa são maiores quando o ambiente de informações contábeis das empresas é inadequado e quando as empresas têm graves problemas de agência, que são medidos por excesso de fluxo de caixa livre. Gupta et al. (2021) verificaram que a educação financeira e técnica do gestor afeta a sensibilidade do investimento ao fluxo de caixa para empresas indianas e Sprenger e Lazarevab (2021) observaram, em uma amostra de empresas russas não listadas em bolsa, que uma melhor proteção aos acionistas diminui a sensibilidade do investimento ao fluxo de caixa.

Diante dessa ausência de entendimento consolidado, o estudo e a análise da relação entre os FCLs e os níveis de Governança Corporativa podem contribuir para a melhor compreensão das dinâmicas das estruturas organizacionais no mercado de capitais brasileiro, além de fornecer subsídios para a tomada de decisões de investimentos pelos participantes desse mercado. Consequentemente, a questão que norteia esta pesquisa pode ser transcrita conforme a seguir: Qual é a relação entre os fluxos de caixa livres das organizações e os diferentes níveis de Governança Corporativa presentes no mercado acionário brasileiro?

Assim, o objetivo deste estudo foi analisar a relação entre os FCL e os diferentes níveis de Governança Corporativa presentes no mercado acionário brasileiro, no período de 2010 a 2018.

A justificativa deste trabalho pauta-se na relevância em analisar, em um mercado acionário sujeito a imperfeições, fatores que possam afetar decisões sobre o nível de manutenção de caixa das empresas, mais especificamente ao avaliar como os mecanismos de Governança Corporativa relacionam-se com a teoria dos FCLs, num contexto de potencial conflito de interesses. Nesse sentido, a pesquisa traz resultados importantes que tornam-se informações úteis para auxiliar o processo decisório dos diversos stakeholders que, por sua vez, são representados por acionistas, investidores, empresas, clientes e fornecedores.

Desta forma, contribui-se ao subsidiar, com achados empíricos, o processo de tomada de decisão, além de fomentar esse tipo de discussão para a consecução de mais pesquisas. Destacase, ainda, que ao investigar o mercado brasileiro, marcado por uma característica de concentração acionária, o estudo ganha distinção em relação aos trabalhos precedentes, o que traz uma originalidade no cenário nacional. Ademais, este estudo também se diferencia dos anteriores ao explorar de que forma os diferentes níveis de Governança Corporativa, diferenciados pelos segmentos de listagem da $[\mathrm{B}]^{3}$, podem estar relacionados com os FCLs das organizações.

\section{REVISÃO DA LITERATURA}

\subsection{Teoria dos Fluxos de Caixa Livres}

Conforme ressalta Lerner (2019), é interessante para os acionistas saberem qual o montante de recursos disponíveis a eles no futuro, por isso, existe o modelo do FCL. Assim, Modigliani e Miller (1958) argumentam que as decisões de investimento são baseadas no FCL das organizações e, na ausência deste, os investimentos serão financiados com dívida ou patrimônio. Uma empresa pode investir mais quando o fluxo de caixa é alto, porque os fundos internos podem ser menos dispendiosos do que os fundos externos e os gerentes podem gastar mais (Yeo, 2018). Por sua vez, Keynes (1936) destaca que as organizações mantêm reservas de excesso de caixa em preparação para manter níveis ótimos de investimento durante períodos negativos de liquidez, denominado como motivo de precaução para manter caixa.

Investimentos insuficientes ou o excesso de investimentos podem afetar o valor de uma empresa. Sabe-se que o valor de uma entidade engloba vários componentes, tais como imóveis, direitos, equipamentos, marcas e caixa. Desses componentes, apenas o caixa pode ser usado livremente pelos gestores, nominado de FCL. A utilização ou o investimento eficiente de ativos 
aumentará o valor da empresa, enquanto a utilização ineficaz de ativos diminuirá o valor da empresa (Yeo, 2018).

Segundo Jensen (1986), a Teoria dos Fluxos de Caixa Livres aborda as operações efetuadas pelos gestores que reduzem o caixa livre ao fazer investimentos que devem possuir valor presente líquido positivo em relação ao custo de capital da organização. Ainda, o autor aponta que a administração tem o potencial de desperdiçar o FCL positivo, investindo em projetos não rentáveis e de baixos retornos para as organizações.

A teoria da agência, segundo Ferreira, Ferreira, Lamounier e Avelar (2021), fornece arcabouço para a Teoria dos FCF, que, em sua base, aborda o conflito de interesses entre os gestores e os acionistas no que tange ao uso discricionário dos recursos da firma para seu próprio benefício. Assim, Lang, Stulz e Walkling (1991) esclarecem que, consoante à teoria dos FCLs, em empresas com poucas alternativas de investimento, os custos de agência se destacam e, portanto, os gestores tendem a investir em alternativas que apresentem um valor presente líquido negativo, o que não acarreta em um aumento do valor da empresa.

Nessa mesma linha, Lerner e Victor (2020) reforçam que, se há custos de agência em determinada firma, existem perdas residuais e a empresa estaria deixando de aumentar seu desempenho econômico. Desse modo, se há FCL utilizados de forma ineficiente, consequentemente, há custos de agência e o desempenho econômico tende a diminuir. Mensurar o que seria o FCL normal ou saudável e o FCL excessivo é um desafio, pois o que pode ser excesso para uma companhia pode não ser para outra, dadas determinadas particularidades como tamanho, endividamento, setor (Lerner \& Victor, 2020) ou mesmo níveis de governança corporativa.

\subsection{Governança Corporativa no Contexto da Teoria da Agência}

Tendo em vista a relação de agência, definida por Jensen e Meckling (1976) como um contrato sob o qual o principal emprega o agente para exercer em seu nome atividades que deleguem algum poder de decisão a este último, cria-se um ambiente carregado de perturbações. Ou seja, essa separação entre o acionista e o gestor desenvolve um ambiente complexo, propício ao surgimento do problema da assimetria de informação, no qual o agente (gestor) tem uma vantagem informacional sobre o principal (acionista), podendo agir de acordo com seus próprios interesses em detrimento do bem estar do acionista (Macho-Stadler \& Pérez-Castrillo, 2001).

Jensen e Meckling (1976) destacam que quando ambas as partes, agente e principal, são maximizadores de utilidade, há motivações para acreditar que o agente nem sempre tomará decisões em prol dos interesses do principal. Não obstante, o principal poderá adotar mecanismos de incentivos para o agente, com vistas a minimizar atividades irregulares deste último, o que lhe trará custos de monitoramento. Em sinergia, Lambright (2009), ao contextualizar os pressupostos da Teoria da Agência, destaca que os desdobramentos dos problemas de agência podem ser compreendidos por meio dos conceitos de seleção adversa e risco moral.

A seleção adversa ocorre quando o mercado de seu posto não pode diferenciar o tipo ou a qualidade dos bens da outra parte, o que deriva da existência de uma informação privilegiada para uma das partes antes da efetivação do contrato. Já o risco moral ocorre quando a ação do agente não é verificável, não permitindo ao principal verificar as ações do agente (Klann, Gomes, Greuel \& Bezerra, 2014).

Com base nesses pressupostos, Jensen e Meckling (1976) argumentam que é impossível para o principal ou para o agente manter a relação de agência sem custos, com vistas a garantir que o agente tomará sempre decisões de nível ótimo considerando o ponto de vista do principal. Com vistas a minimizar tais conflitos, as empresas adotam a prática de mecanismos que possam monitorar os agentes, através da Governança Corporativa.

Desse modo, o "desenvolvimento da governança corporativa tem sido marcado pela busca pela minimização de conflitos entre acionistas e executivos" (Oliveira \& Fontes Filho, 2021, p. 
511). Shleifer e Vishny (1997) elucidam que a Governança Corporativa lida com as maneiras pelas quais os fornecedores de recursos garantem que obterão para si o retorno sobre seu investimento. Num contexto mais normativo, o Instituto Brasileiro de Governança Corporativa (IBGC) elucida que a "Governança Corporativa é o sistema pelo qual as empresas e demais organizações são dirigidas, monitoradas e incentivadas, envolvendo os relacionamentos entre sócios, conselho de administração, diretoria, órgãos de fiscalização e controle e demais partes interessadas" (IBGC, 2019).

Ainda de acordo com o IBGC (2019), as boas práticas de Governança Corporativa tendem a convergir princípios em recomendações objetivas, de forma a alinhar interesses e com vistas a otimizar o valor da empresa. No tocante aos seus princípios, Lodi (2000) assim os apresenta: i) fairness: senso de justiça e equidade para com os acionistas minoritários contra transgressões de majoritários e gestores; ii) disclosure: chamada de transparência, compreende dados acurados, registros contábeis fora de dúvida e relatórios entregues nos prazos combinados; iii) accountability: denominada de prestação de contas, refere-se à prestação de contas por parte dos que tomam as decisões de negócios; e iv) compliance: cumprimento e obediência às leis do país.

Conforme a [B] ${ }^{3}$ (2019), no Brasil, os segmentos especiais de listagem são caracterizados em: a) Novo Mercado: lançado no ano 2000, o Novo Mercado estabeleceu desde sua criação um padrão de Governança Corporativa altamente diferenciado; b) Nível 1: as empresas listadas no segmento Nível 1 devem adotar práticas que favoreçam a transparência e o acesso às informações pelos investidores. Para isso, divulgam informações adicionais às exigidas em lei como, por exemplo, um calendário anual de eventos corporativos; c) Nível 2: é similar ao Novo Mercado, porém com algumas exceções. As empresas listadas têm o direito de manter ações preferenciais; e d) Bovespa mais: idealizado para empresas que desejam acessar o mercado de forma gradual, esse segmento tem como objetivo fomentar o crescimento de pequenas e médias empresas via mercado de capitais.

Os mecanismos de governança corporativa, segundo destacam Hedlund et al. (2021), se configuram como uma importante ferramenta estratégica nas organizações, contribuindo para a redução dos conflitos de agência e para o desenvolvimento de fortes relacionamentos entre todos os interessados na firma. Assim, práticas de governança eficazes podem ajudar a controlar o dilema que existe entre gestores e acionistas (Sehrawat et al., 2019). Com os mecanismos de accountability, transparência e supervisão, as melhores práticas de governança corporativa podem aumentar a comunicação e a confiança dos acionistas nos gestores, o que significa que a compreensão desses mecanismos pode ajudar a reduzir o problema de agência entre proprietários e gestores (Huu Nguyen, Thuy Doan \& Ha Nguyen, 2020).

Desse modo, espera-se que empresas com maiores índices de qualidade de Governança Corporativa ofereçam uma proteção mais consistente aos seus investidores, por meio de uma gestão profissional, responsável, transparente e justa para acionistas, executivos e terceiros, levando-se em conta que boas práticas de Governança Corporativa permitem minimizar os impactos de comportamentos oportunistas dos gestores (Silva, Caixe \& Krauter, 2019). Em completude, Dylewski (2010) destaca a hipótese de que uma gestão não comprometida com boas práticas de Governança Corporativa apresentaria maiores níveis de caixa, sendo uma relação esperada.

\subsection{Fluxos de Caixa Livres e Governança Corporativa}

Uma série de estudos têm sido realizados acerca das temáticas da teoria da agência, da teoria dos fluxos de caixa e da Governança Corporativa e suas inter-relações. A maioria das pesquisas visa levantar evidências sobre a relação de elementos da Governança Corporativa. Esta 
subseção apresenta um resumo dos resultados de algumas pesquisas acadêmicas já realizadas com enfoque na teoria de FCL e Governança Corporativa.

Dittmar e Mahrt-Smith (2007) investigaram se a Governança Corporativa afetava o valor da empresa, comparando o valor e o uso de reservas de caixa em empresas bem e mal gerenciadas. Para isso, os autores realizaram a estimação de uma regressão por mínimos quadrados ordinários (OLS) com erros padrão robustos de todas as empresas de capital aberto dos Estados Unidos, no período de 1990 a 2003. Encontraram evidências de que elevados níveis de Governança Corporativa dobram as sobras de caixas, por outro lado, as empresas com poucas práticas de Governança Corporativa dissipam o dinheiro rapidamente, ocasionando a redução significativa do desempenho operacional. Assim, a governança tem uma influência maior sobre o uso, e não sobre a acumulação, de reservas de caixa. Isso implica que a governança afeta as decisões operacionais e de investimento (como usar caixa) mais do que as decisões de financiamento relacionadas à política de caixa (quanto dinheiro acumular).

McKnight e Weir (2009) examinaram a relação entre a Governança Corporativa e os custos de agência, medidos pela relação entre as vendas e o total de ativos, a interação de fluxos de caixa livres e perspectivas de crescimento e o número de aquisições. A amostra compreendeu empresas cotadas publicamente no Reino Unido, no período de 1996 a 2000. Os resultados empíricos evidenciaram que o aumento da propriedade do conselho e a dívida reduzem os custos de agência. No entanto, as mudanças nas estruturas dos Conselhos têm impacto nos custos de agência e ter um comitê de nomeação aumenta, ao invés de reduzir, os custos da agência.

Dylewski (2010) estudou os determinantes dos níveis de ativos líquidos de empresas abertas do Brasil, Argentina, Chile, México e Peru no período de 1995 a 2009, dado o cenário de tendência crescente de acúmulo de ativos líquidos. A autora encontrou evidências de que empresas com maiores oportunidades de crescimento, maior tamanho, maior nível de pagamento de dividendos e maiores níveis de lucratividade acumulam mais caixa na maior parte dos países analisados. Da mesma forma, empresas com maiores níveis de investimento em ativo imobilizado, maior geração de caixa, maior volatilidade do fluxo de caixa, maior alavancagem e maior nível de capital de giro apresentam menor nível de acúmulo de ativos líquidos. Ademais, constatou-se, também, que empresas com maiores níveis de Governança Corporativa possuem mais caixa, estando contrária à hipótese de que uma administração mais comprometida mantenha menor nível de liquidez.

Chi e Lee (2010) examinaram a relação entre a Governança Corporativa e o valor da empresa condicionada ao nível de FCL disponível para os administradores. Foi feita a análise de empresas norte americanas nos anos 1990-2004, empregando o método de regressão de Fama e MacBeth (1973), que envolve a execução de regressões anuais do valor da empresa em variáveis de governança simultâneas, calculando a média das séries temporais das estimativas do coeficiente e inferindo a significância estatística com base nos erros padrão das séries temporais. Os autores encontraram evidências de que a Governança Corporativa afeta o valor da empresa de maneira diferente, dependendo se a empresa possui FCL alto ou baixo. Especificamente, o valor da empresa aumenta com melhores níveis de governança entre as entidades de alto FCL, enquanto o efeito da governança é menor ou insignificante entre as empresas de baixo FCL.

Por sua vez, Wang (2010) investigou como o FCL estava associado aos custos de agência e influenciava o desempenho da empresa. Para testar as hipóteses, os dados foram baseados em todas as empresas listadas publicamente na Bolsa de Valores de Taiwan, no período de 2002 a 2007. O autor identificou que os custos de agência estão associados negativamente e significativamente ao desempenho da empresa, enquanto o FCL está relacionado positivamente e significamente ao desempenho da entidade. O primeiro resultado apóia a teoria da agência, enquanto o segundo é inconsistente com a hipótese de FCL. 
Silva, Caixe e Krauter (2019) investigaram a sensibilidade do investimento ao fluxo de caixa para empresas brasileiras listadas na $[\mathrm{B}]^{3}$ com diferentes níveis de governança corporativa, no período de 2006 a 2015. Os resultados evidenciaram que a qualidade da governança corporativa influenciou a sensibilidade investimento-fluxo de caixa, de modo que essa relação foi negativa e significante apenas para as empresas com pior governança. Tal achado pode ser entendido como um indício de que tais empresas buscam aumentar suas reservas de caixa e reduzir investimentos por motivos relativos a restrições financeiras e/ou a problemas de agência.

Zhang (2020) buscou relacionar a Governança Corporativa aos custos de agência dos Fluxos de Caixa Livres nas aquisições domésticas dos Estados Unidos. Os achados indicaram que a qualidade da Governança Corporativa afeta indiretamente os resultados da fusão ao mitigar o problema de agência associado à retenção, ao invés do uso, de fluxos de caixa livres. Já Waly, Sasongko e Achyani (2021) analisaram o efeito do fluxo de caixa livre, da divulgação de informações sobre Gestão de Riscos Corporativos e do relatório de sustentabilidade sobre o valor das empresas listadas no Jakarta Islamic Index durante o período 2015-2019, tendo a boa governança corporativa como uma variável moderadora. Os resultados evidenciaram que nenhuma das três variáveis analisadas - Fluxo de Caixa Livre, divulgação de informações sobre Gestão de Riscos Corporativos e do relatório de sustentabilidade - tiveram efeito sobre o valor das empresas da amostra.

Sprenger e Lazarevab (2021) investigaram, em uma amostra de empresas russas não listadas em bolsa, como a Governança Corporativa afetava as restrições de financiamento, medidas pela sensibilidade do investimento ao fluxo de caixa. O principal resultado encontrado foi que uma melhor proteção aos acionistas diminui a sensibilidade do investimento ao fluxo de caixa, especialmente em empresas com um controlador externo. Em contrapartida, esse efeito não foi verificado para a transparência, o que pode ser parcialmente explicado pela ameaça de aquisições hostis.

Com base nos estudos apresentados, a relação entre o FCL e a Governança Corporativa tem sido abordada em diversos contextos. Dittmar e Mahrt-Smith (2007) e Chi e Lee (2010) focaram na relação da Governança Corporativa afetando o valor da empresa, encontrando evidências, respectivamente, que elevados níveis de governança dobram as sobras de caixa e que o valor da empresa aumenta com melhores níveis de governança. Já Dylewski (2010) encontrou achados que sugerem que quanto maior o nível de Governança Corporativa, maior tende a ser o nível de ativo líquido das empresas. Por fim, Wang (2010) e McKnight e Weir (2009) focaram na questão do custo de agência, com achados, respectivamente, de que os custos de agência se relacionam negativamente ao desempenho da empresa e de que mudanças estruturais do Conselho agregam pouco efeito nos custos de agência.

\section{METODOLOGIA}

\subsection{Classificação da Pesquisa, População, Amostra e Coleta de Dados}

Quanto à classificação da pesquisa, este estudo adotou a categorização apresentada em Raupp e Beuren (2008), sendo classificado como descritivo e explicativo, documental e quantitativo.

A população deste estudo englobou todas as empresas brasileiras de capital aberto listadas na $[\mathrm{B}]^{3}$ no período de 2010 a 2018 , perfazendo um total de nove anos. $\mathrm{O}$ ano inicial do período escolhido se justifica em função da convergência às normas internacionais de contabilidade, que começaram a vigorar no Brasil com a publicação das Leis n. ${ }^{\circ}$ 11.638/07 e 11.941/2009, além dos Pronunciamentos Técnicos emanados pelo Comitê de Pronunciamento Contábeis (CPC) (Gelbecke, Santos, Iudicíbus \& Martins, 2018). Assim, a partir do ano de 2010, as demonstrações financeiras publicadas pelas entidades começaram a seguir um padrão internacional, seguindo 
critérios de padronização e comparabilidade. Por sua vez, o ano final de 2018 se justifica em virtude de ser o último ano com dados anuais encerrados e disponibilizados na data de realização desta pesquisa.

O processo de coleta de dados foi realizado por intermédio do software Economática ${ }^{\circledR}$. Desta forma, tornou-se possível acessar todas as demonstrações financeiras das empresas brasileiras listadas na $[\mathrm{B}]^{3}$. Por conseguinte, foram excluídas da população inicial as empresas caracterizadas como instituições financeiras, pois estas possuem particularidades, com demonstrações contábeis e planos de contas diferentes das demais entidades.

Outro critério adotado foi o de excluir da população inicial todas as entidades que não apresentaram informação da receita líquida em todos os anos. A escolha por essa tratativa se justifica por considerar que empresas que não apresentaram tal informação em algum dos anos de análise não tenham desenvolvido suas operações naquele período. O referido critério também foi adotado por Avelar, Cunha, Boina e Souza (2015), que justificaram que a receita líquida representa as receitas advindas das atividades operacionais da empresa.

Cabe destacar que o processo de amostragem é não probabilístico, pois parte-se de um universo naturalmente restrito, com exclusão de entidades que não apresentaram dados suficientes, o que faz com que os resultados não possam ser generalizados a toda a população de empresas brasileiras. A amostra final desta pesquisa foi composta por 212 companhias, sendo que a maior concentração está nas empresas dos setores econômicos Consumo Cíclico, Bens Industriais e Utilidade Pública, que representam, respectivamente, 30\%, 20\% e 20\% do total da amostra. Ainda, a amostra do estudo foi composta de forma desbalanceada.

\subsection{Variáveis Analisadas}

A variável dependente analisada neste estudo se refere aos FCLs, representada pelo código FCL. A sua metodologia de cálculo é elucidada pela Equação 1:

$$
F C L=N O P A T+D E P-C A P E X-I N V C A P
$$

Em que:

FLC = Fluxo de caixa livre;

NOPAT $=$ Net Operating Profits Less Adjusted Tax - Lucro Operacional menos Impostos Ajustados;

DEP = Depreciação;

CAPEX = Capital Expenditure - Despesas de Capital;

INVCAP = Investimento em Capital de Giro.

Posteriormente, com vistas a minimizar a variabilidade das observações encontradas nessa variável, dada a diversidade da amostra, aplicou-se o logaritmo natural ao valor apresentado.

As variáveis independentes do modelo proposto, incorporadas com o intuito de estabelecer relações com a variável dependente, foram as seguintes: i) Níveis de Governança Corporativa que, na amostra final, foram representadas pelos níveis: Tradicional, Novo Mercado, Nível 1 e Nível 2; ii) tamanho da empresa, representado pelo ativo total; iii) rentabilidade, sendo a proxy adotada o ROE (Retorno sobre o Patrimônio Líquido); e iv) taxa de Crescimento, aferida pela variação da receita líquida. É válido destacar que a inclusão das variáveis se origina na teoria dos FCLs apresentada por Jensen (1986), assim como buscou-se, também, encontrar na literatura outros autores que já pesquisaram as citadas variáveis, com vistas a corroborar as relações esperadas neste estudo. Na Tabela 1, elencam-se as variáveis anteriormente mencionadas. 
Tabela 1

Variáveis explicativas do modelo

\begin{tabular}{|c|c|c|c|}
\hline Variável & Proxy & Fonte & Relação Esperada \\
\hline $\begin{array}{l}\text { Nível de Governança } \\
\text { Novo Mercado } \\
\text { (NOVMER) }\end{array}$ & $\begin{array}{c}\text { Variável dicotômica, assume valor } 1 \text { se } \\
\text { pertencente ao segmento Novo Mercado e } 0 \text { caso } \\
\text { contrário }\end{array}$ & $\begin{array}{l}\text { Jensen }(1986) \\
\text { Dylewski } \\
\text { (2010). }\end{array}$ & - \\
\hline $\begin{array}{l}\text { Nível de Governança } \\
\text { Nível } 1 \\
\text { (NIV1) }\end{array}$ & $\begin{array}{c}\text { Variável dicotômica, assume valor } 1 \text { se } \\
\text { pertencente ao segmento Nível } 1 \text { e } 0 \text { caso } \\
\text { contrário }\end{array}$ & $\begin{array}{l}\text { Jensen }(1986) \\
\text { Dylewski } \\
\text { (2010). }\end{array}$ & - \\
\hline $\begin{array}{l}\text { Nível de Governança } \\
\text { Nível } 2 \\
\text { (NIV2) }\end{array}$ & $\begin{array}{c}\text { Variável dicotômica, assume valor } 1 \text { se } \\
\text { pertencente ao segmento Nível } 2 \text { e } 0 \text { caso } \\
\text { contrário }\end{array}$ & $\begin{array}{l}\text { Jensen }(1986) \\
\text { Dylewski } \\
\text { (2010). }\end{array}$ & - \\
\hline $\begin{array}{l}\text { Tamanho da Empresa } \\
\text { (TAM) }\end{array}$ & Ln do Ativo Total & Jensen (1986). & + \\
\hline $\begin{array}{l}\text { Retorno sobre o } \\
\text { Patrimônio Líquido } \\
\text { (ROE) }\end{array}$ & $\frac{\text { Lucro Líquido }}{\text { Patrimônio Líquido médio }}$ & Jensen (1986). & - \\
\hline $\begin{array}{l}\text { Taxa de Crescimento } \\
\text { (TAXCRESC) }\end{array}$ & $\frac{(\text { Receita Líquida })_{t}-(\text { Receita Líquida })_{t_{-1}}}{(\text { Receita Líquida })_{t_{-1}}}$ & $\begin{array}{c}\text { Jensen e } \\
\text { Meckling } \\
\quad(1976) ; \\
\text { Jensen (1986). }\end{array}$ & - \\
\hline
\end{tabular}

Fonte: Elaborada pelos autores

Conforme detalhado na Tabela 1, os níveis de Governança Corporativa foram representados por variáveis dummies, ou seja, variáveis de natureza dicotômica, que assumem valor 0 na ausência do atributo considerado pela variável e valor 1 na presença do atributo (Gujarati \& Porter, 2011). Os níveis de governança presentes foram: Tradicional, Novo Mercado, Nível 1 e Nível 2. Dessa forma, adotou-se como controle o nível Tradicional, o que permitiu a inclusão de três variáveis dummies.

A primeira dummy foi representada pelo código NOVMER, em que assume valor 1 caso a empresa pertença ao nível Novo Mercado e 0 caso contrário. A segunda dummy, de código NIV1, representou o Nível 1, sendo que empresas pertencentes ao respectivo nível assumiram valor $1 \mathrm{e}$ 0 caso contrário. A terceira e última dummy recebeu o código NIV2 e foi representada pelo Nível 2 que, por sua vez, assumiu valor 1 caso a empresa pertença a esse nível e 0 caso contrário.

A inclusão das variáveis relacionadas à Governança Corporativa fundamenta-se no estudo de Jensen (1986), que elucida que empresas com excesso de caixa incorrem em custos de agência, dado que os agentes (gerentes) optam por não pagar dividendos aos acionistas, mesmo na ausência de projetos rentáveis de investimento. Partindo dessa premissa, a adoção de práticas de Governança Corporativa poderia minimizar esse conflito entre agente versus principal. Dylewski (2010) abordou o nível de governança das empresas participantes do Índice de Ações com Governança Corporativa da BMF\&Bovespa (IGC), encontrando relação negativa com a variável liquidez das empresas, sendo representada pelos ativos líquidos. Em sinergia, a relação esperada neste estudo é negativa, ou seja, empresas com boas práticas de Governança Corporativa tenderiam a apresentar menores FCLs.

As demais variáveis independentes foram de natureza quantitativa. Assim, a variável tamanho da empresa foi representada pelo código TAM e foi obtida pelo cálculo do logaritmo natural do seu ativo total. A utilização dessa variável pauta-se no trabalho de Jensen (1986), o qual 
destaca que empresas consideradas como maduras, por já terem concretizado todos os investimentos necessários para seu crescimento no passado, tendem a apresentar maior valor de ativos. Assim, espera-se uma relação positiva com a variável dependente.

Em completude, para representar a variável independente Rentabilidade, adotou-se como proxy o retorno sobre o patrimônio líquido, também conhecido como ROE, sendo essa a descrição da variável no modelo. Partindo de Jensen (1986), que infere que os gestores optam por investir em projetos não rentáveis em detrimento de remunerar os acionistas, espera-se que quanto menor a rentabilidade, menor será o FCL.

Por fim, a última variável independente foi a Taxa de Crescimento, representada pelo código TAXCRESC. O cálculo desta variável foi obtido pela variação da receita líquida em relação ao período anterior. A teoria apresentada por Jensen (1986) contextualiza que empresas em fase de crescimento, que ainda não atingiram um estágio de amadurecimento, tendem a apresentar um menor volume de FCLs. Sob essa ótica, espera-se encontrar uma relação negativa com a variável dependente. Ademais, Jensen e Meckling (1976) corroboram que as empresas que estão mais propícias a gerarem maiores valores de FCLs são representadas por empresas situadas em setores mais maduros.

\subsection{Modelo Econométrico e Tratamento dos Dados}

Dado que esta pesquisa lida com dados que variam no tempo e no espaço, constitui-se um painel de observações. Sob esse aspecto, Gujarati e Porter (2011) destacam que nos dados em painel a mesma unidade de corte transversal (uma família, uma empresa, um estado) é acompanhada ao longo do tempo. Desta forma, o modelo econométrico proposto neste estudo encontra-se detalhado na Equação 2.

$$
\begin{aligned}
& F C L_{i t}=\beta_{0}+\beta_{1} \times N O V M E R_{i t}+\beta_{2} \times N I V 1_{i t}+\beta_{3} \times N I V 2_{i t}+\beta_{4} \times T A M_{i t}+\beta_{5} \times R O E_{i t}+ \\
& \beta_{6} \times T_{A X C R E S C}+c i+\varepsilon_{i t}
\end{aligned}
$$

Em que:

$\mathrm{FCL}_{\mathrm{it}}=$ Fluxos de Caixa Livres;

$\beta_{0}=$ Intercepto do modelo (constante);

$\beta_{1}, \beta_{2}, \beta_{3}, \beta_{4}, \beta_{5}$ e $\beta_{6}=$ Coeficientes angulares do modelo;

NOVMER ${ }_{\text {it }}=$ Nível de Governança Novo Mercado. Assume valor 1 quando a empresa pertencer ao Novo Mercado e 0 caso contrário;

$\mathrm{NIV}_{1}$ it $=$ Nível de Governança Nível 1 . Assume valor 1 quando a empresa pertencer ao Nível $1 \mathrm{e}$ 0 caso contrário;

$\mathrm{NIV} 2_{\mathrm{it}}=$ Nível de Governança Nível 2. Assume valor 1 quando a empresa pertencer ao Nível 2 e 0 caso contrário;

$\mathrm{TAM}_{\mathrm{it}}=$ Tamanho da empresa;

$\mathrm{ROE}_{\mathrm{it}}=$ Retorno sobre o patrimônio líquido;

TAXCRESC $_{\text {it }}=$ Taxa de crescimento;

$c_{i}=$ efeito individual específico não observável, que difere entre as unidades, e é invariante no tempo;

$\varepsilon_{\mathrm{it}}=$ Erro usual da regressão.

Os modelos de dados em painel podem ser apresentados em três principais grupos: i) Modelo MQO para dados empilhados (pooled data); ii) Modelo de Efeitos Fixos (MEF); e iii) Modelo de Efeitos Aleatórios (MEA). No modelo pooled, estima-se uma regressão desprezando a 
natureza de corte transversal e de séries temporais, trabalhando-se com dados empilhados. No MEF, o intercepto do modelo de regressão pode diferir entre os indivíduos, devido ao fato de que cada indivíduo, ou unidade de corte transversal, pode ter características especiais próprias. Já o MEA pressupõe que os valores de intercepto sejam extraídos aleatoriamente de uma população bem maior com um valor médio constante (Gujarati \& Porter, 2011).

Foram realizados todos os testes estatísticos para se chegar ao modelo final a ser apresentado pelo estudo, com a definição da abordagem que melhor atende as características da amostra. Os testes realizados para escolha do modelo foram adotados conforme balizado na literatura, tais como o teste de Chow, o teste de Breusch-Pagan (BP) e o teste de Hausman (Baltagi, 2005; Gujarati \& Porter, 2011). Ademais, compete informar que foram observados todos os pressupostos para a validação do modelo, tais como: ausência de multicolinearidade, correta especificação do modelo, normalidade dos resíduos, ausência de autocorrelação serial e resíduos homocedásticos. Os dados foram tratados e tabulados por intermédio do software STATA $14.0^{\circledR} \mathrm{e}$ do software Microsoft Excel ${ }^{\circledR}$ (MS-Excel) 2019.

\section{ANÁLISE DOS RESULTADOS}

\subsection{Estatísticas Descritivas}

Após a definição da amostra final, buscou-se apresentar as estatísticas descritivas acerca das variáveis analisadas neste estudo. Em relação aos segmentos de listagem na [B] $]^{3}$, observou-se a predominância de empresas aderentes ao Novo Mercado, seguidas de empresas pertencentes ao segmento Tradicional, Nível 1 e Nível 2, sendo, respectivamente, representadas por 45\%, 39\%, $9 \%$ e $6 \%$ do total da amostra. Já na Figura 1, apresenta-se o histórico do FCL do período de 2010 a 2018 para os níveis de Governança Corporativa Tradicional, Nível 1, Nível 2 e Novo Mercado.

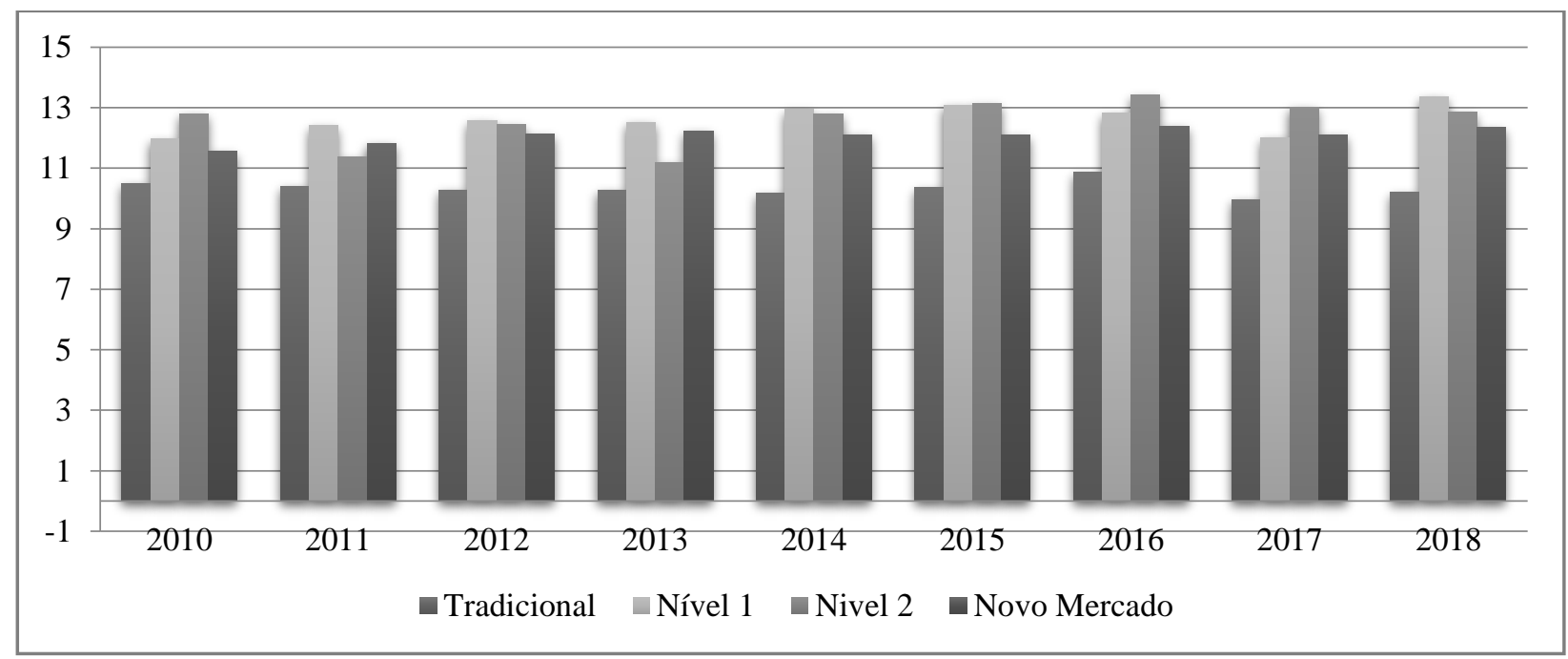

Figura 1. FCL por Nível de Governança Corporativa

Fonte: Dados da pesquisa.

Com base nos dados apresentados na Figura 1, identifica-se como característica das empresas abertas brasileiras presentes na amostra o fato de apresentarem menores FCLs em empresas que não se enquadram em nenhum dos níveis de Governança Corporativa e maiores FCLs para os Níveis 1 e 2, com uma pequena redução no nível Novo Mercado. Isso demonstra que existe uma tendência de crescimento de FCL até o penúltimo nível de Governança Corporativa e um leve declínio no ultimo nível. Neste estudo, tendo como base a hipótese de Jensen (1986) de 
que a Governança Corporativa reduziria o FCL, esperava-se que empresas que não se enquadrassem em nenhum dos níveis de Governança apresentassem os maiores FCLs, o que não foi o caso. No entanto, resultados mais assertivos poderão ser observados apenas após a estimação do modelo de regressão, temática do tópico seguinte.

Na Tabela 2, apresenta-se a estatística descritiva dos dados, demonstrando as médias, os desvios-padrão, os valores mínimos e máximos, coeficiente de variação, grau de assimetria e quartis das variáveis quantitativas empregadas no estudo.

Tabela 2

Estatística Descritiva das Variáveis para Amostra com base em novembro de 2019

\begin{tabular}{l|c|c|c|c|c|c|c}
\hline Descrição & FCL & NOVMER & NIV1 & NIV2 & TAM & ROE & TAXCRESC \\
\hline Observações & 1105 & 1908 & 1908 & 1908 & 1908 & 1707 & 1899 \\
\hline Mínimo & 0 & 0 & 0 & 0 & 8,69 & $-5451,53$ & $-1,33$ \\
\hline Máximo & 18,09 & 1 & 1 & 1 & 20,62 & 7500 & 109,77 \\
\hline Média & 11,56 & 0,453 & 0,094 & 0,061 & 14,742 & 0,082 & 0,219 \\
\hline Desvio Padrão & 2,16 & 0,498 & 0,292 & 0,24 & 1,839 & 245,402 & 2,834 \\
\hline Coeficiente de Variação & 0,19 & 1,1 & 3,099 & 3,914 & 0,125 & 3004,6 & 12,916 \\
\hline Assimetria & $-0,65$ & 0,19 & 2,776 & 3,657 & $-0,09$ & 10,048 & 33,154 \\
\hline Q25 & 10,27 & 0 & 0 & 0 & 13,525 & 0,35 & $-0,03$ \\
\hline Q50 & 11,79 & 0 & 0 & 0 & 14,81 & 8,53 & 0,08 \\
\hline Q75 & 12,98 & 1 & 0 & 0 & 16 & 16,92 & 0,19 \\
\hline
\end{tabular}

Fonte: Dados da pesquisa.

Com base nos dados da Tabela 2, observa-se que as variáveis do estudo possuem certa linearidade e, pela análise dos desvios padrão, dos valores máximos e mínimos, pode-se inferir que os dados estão organizados em torno da média homogeneamente. A única variável que foge um pouco a esta configuração é a taxa de crescimento, que apresenta média de 0,219 , valor mínimo de $-1,33$ e máximo de 109,77, demonstrando que há uma certa concentração de valores negativos abaixo da média.

\subsection{Estatística Inferencial}

Com o propósito de identificar o modelo mais adequado para a apresentação e a discussão dos resultados, foram realizados testes estatísticos para a validação do modelo final. O nível de significância adotado neste estudo foi de 5\%. Na Tabela 3, apresenta-se uma síntese dos resultados dos testes aplicados.

Tabela 3

Testes de validação do modelo com dados com base em novembro de 2019

\begin{tabular}{lcccc} 
Critério analisado & Teste utilizado & VIF & Valor-P & Resultado \\
\hline $\begin{array}{l}\text { Ausência de } \\
\text { Multicolinearidade }\end{array}$ & $\begin{array}{c}\text { Teste de fator de inflação da } \\
\text { variância (VIF) }\end{array}$ & 1,24 & - & $\begin{array}{c}\text { Ausência de } \\
\text { multicolinearidade }\end{array}$ \\
Forma funcional adequada & Teste RESET & - & 0,1209 & Forma funcional adequada. \\
Modelo pooled versus MEF & Teste de Chow & - & 0,0000 & MEF preferível \\
Modelo pooled versus MEA & Teste de Breusch-Pagan & - & 0,0000 & MEA preferível
\end{tabular}




\begin{tabular}{lcccc}
\hline Critério analisado & Teste utilizado & VIF & Valor-P & Resultado \\
\hline MEA versus MEF & Teste de Hausman & - & 0,6697 & MEA preferível \\
Ausência de Autocorrelação & Teste de Wooldridge & - & 0,0084 & Presença de autocorrelação \\
Ausência de & Teste de Likelihood-Ratio & - & 0,0000 & $\begin{array}{c}\text { Presença de } \\
\text { Heterocedasticidade }\end{array}$ \\
\hline
\end{tabular}

Fonte: Dados da pesquisa.

Estimou-se, inicialmente, o modelo pooled e, a partir dos resultados, avaliou-se o pressuposto de ausência de multicolinearidade e se o modelo possuía adequação da forma funcional. O teste de inflação da variância (VIF) foi utilizado para checar se o modelo indicava a presença de forte correlação entre as variáveis explicativas. Fávero, Belfiore, Silva e Chan (2009) elucidam que um VIF acima de 5 pode acarretar em problemas de multicolinearidade. O resultado conjunto ou individual não indicou a presença de multicolinearidade, tendo sido encontrado um VIF médio de 1,24.

Em seguida, checou-se se o modelo apresentava adequada forma funcional. O teste realizado foi o de Ramsey, denominado teste RESET (Regression Specification Error Test). Gujarati e Porter (2011) destacam que uma vantagem do RESET é a sua facilidade de aplicação, pois não exige que se especifique qual é o modelo alternativo. A hipótese nula do teste indica que o modelo não apresenta variáveis relevantes omitidas, exibindo forma funcional adequada. $\mathrm{O}$ resultado encontrado para o teste em questão foi um valor-p de 0,1209 , o que faz com que não seja rejeitada a hipótese nula ao nível de $5 \%$ de significância, indicando a correta especificação do modelo.

O próximo passo consistiu em estimar o modelo considerando efeitos fixos. A partir da sua estimação, foi possível examinar o resultado do Teste de Chow, com vistas a avaliar a utilização do modelo com efeitos fixos versus o modelo pooled. O resultado do valor-p foi de 0,000 , rejeitando-se, assim, ao nível de 5\% de significância, a hipótese nula de utilização do modelo pooled, sendo preferível o MEF.

Na sequência, estimou-se o modelo considerando efeitos aleatórios (MEA) e aplicou-se o teste do Multiplicador de Lagrange (LM), desenvolvido por Breusch e Pagan, para avaliar a utilização do modelo pooled versus o MEA. O valor-p encontrado foi de $0,0000, \operatorname{logo}$, foi rejeitada a hipótese nula de utilização do modelo pooled, sendo preferível o MEA.

Diante desse cenário, com vistas a validar o modelo a ser utilizado, MEF ou MEA, adotouse o teste de Hausman. Johnston e DiNardo (2001) esclarecem que, para a realização do teste de Hausman, deduzem-se dois estimadores com diferentes propriedades, de forma a considerar se o efeito individual específico não observável $\left(\mathrm{c}_{\mathrm{i}}\right)$ e os regressores estão ou não correlacionados. Assim, a hipótese nula aponta que se o $c_{i}$ não é correlacionado com as variáveis explicativas o modelo de efeitos aleatórios deve ser utilizado. O valor-p encontrado foi de 0,6697 , não rejeitando a hipótese nula, logo, o modelo mais indicado é o MEA.

Após a definição do modelo de efeitos aleatórios como o mais adequado para este estudo, testou-se, por intermédio do teste de Wooldridge, se ele não apresentava autocorrelação de primeira ordem, sendo essa a hipótese nula do teste. Ao nível adotado de 5\% de significância, rejeitou-se a hipótese nula, dado o valor-p encontrado de 0,0084, isto é, o modelo apresentou problema de autocorrelação. Nesse caso, Gujarati (2006) sugere que na presença de autocorrelação, o painel seja estimado pelo método de mínimos quadrados generalizados. Dessa forma, estimou-se o modelo pelo método citado, considerando a presença de autocorrelação. 
Em completude, foi verificado se o modelo estimado por mínimos quadrados generalizados apresentava o problema de heterocedasticidade. O teste de Likelihood-Ratio (LR) apresentou um valor-p de 0,0000, o que faz com que seja rejeitada a hipótese nula de homocedasticidade, então o modelo apresentou o problema de heterocedasticidade. Diante desse cenário, dadas as presenças de autocorrelação e heterocedasticidade, estimou-se o modelo final por mínimos quadrados generalizados, assumindo os ajustes para autocorrelação e heterocedasticidade. Na Tabela 4, apresentam-se os resultados encontrados para o modelo final ajustado.

Tabela 4

MEA ajustado para autocorrelação e heterocedasticidade com dados de novembro de 2019

\begin{tabular}{|c|c|c|c|c|}
\hline Covariância(s) estimada(s) & 184 & & N. ${ }^{\circ}$ observações & 980 \\
\hline Autocorrelação(es) estimada(s) & 1 & & N..$^{o}$ grupos & 184 \\
\hline Coeficientes estimados & 7 & & Obs. grupo: & 2 \\
\hline Wald $\left(x^{2}\right)$ & 12565,16 & & méd & 5,3261 \\
\hline Prob $>x^{2}$ & 0.0000 & & máx & 9 \\
\hline FCL & Coef. & Erro Padrão & $\mathbf{Z}$ & $\mathbf{P}>|\mathbf{Z}|$ \\
\hline NOVMER & 0,1084214 & 0,0347126 & 3,12 & 0,002 \\
\hline NIV1 & $-0,0495338$ & 0,0710259 & $-0, .70$ & 0,486 \\
\hline NIV2 & 0,44608052 & 0,0735031 & 6,08 & 0,000 \\
\hline TAM & 0,9260518 & 0,0110428 & 83,86 & 0,000 \\
\hline ROE & 0,0004246 & 0,000082 & 5,18 & 0,000 \\
\hline TAXCRESC & $-0,243468$ & 0,0559229 & $-4,35$ & 0,000 \\
\hline Constante & $-2,013471$ & 0,01533721 & $-13,13$ & 0,000 \\
\hline
\end{tabular}

Fonte: Dados da pesquisa.

Com base na análise dos resultados do modelo final validado, pelo teste de Wald, verificouse a rejeição da hipótese nula de que todos os parâmetros estimados são estatisticamente iguais a zero. No tocante às variáveis explicativas, para analisar a significância individual de cada uma delas, avaliou-se o resultado do valor-p do teste $Z$. Nota-se que a única variável que não apresentou significância, ao nível de $5 \%$, foi a NIV1, que representa as empresas listadas na $[\mathrm{B}]^{3}$ no segmento Nível 1.

As variáveis NOVMERC e NIV2 apresentaram significância estatística e indicaram uma relação positiva com a variável dependente FCL. Tal resultado implica que as empresas que adotam níveis de Governança Corporativa tendem a apresentar maiores FCLs, o que contrapõe o resultado esperado neste estudo, num contexto de mecanismos utilizados para minimizar conflitos de agência. Dylewski (2010) destaca que essa relação possa ser menos aplicável em países em desenvolvimento, visto que o custo de oportunidade de manter recursos em aplicações financeiras em comparação a investimentos produtivos é menor. Ademais, empresas com maiores níveis de governança podem estar comprometidas em otimizar seu nível de alavancagem, além da possibilidade de manter um nível de "colchão" de liquidez para enfrentar momentos de crise.

Estudos como os de Silva et al. (2019) e de Sprenger e Lazarevab (2021), que analisaram especificamente a sensibilidade do investimento ao fluxo de caixa, também podem auxiliar no entendimento dos resultados desta pesquisa. Segundo Silva et al. (2019), uma possível explicação para o impacto positivo do fluxo de caixa no investimento, atrelada às práticas de governança corporativa, é a de que os gestores tenderiam a superinvestir os fluxos de caixa excedentes ao buscarem seus benefícios privados, de modo que melhores práticas de governança corporativa reduziriam, também, a influência positiva do fluxo de caixa no investimento (Francis, Hasan, Song \& Waisman, 2013). 
Os resultados empíricos observados por Silva et al. (2019) apontaram que as empresas com piores práticas de governança têm sensibilidade negativa do investimento ao fluxo de caixa, fato que pode ser atribuído à vontade dessas companhias em elevarem suas reservas de caixa por motivos associados a restrições financeiras e/ou a problemas de agência. Já Sprenger e Lazarevab (2021) observaram que uma melhor proteção aos acionistas, o que está associado a mecanismos de Governança Corporativa, diminui a sensibilidade do investimento ao fluxo de caixa.

Neste estudo, cuja variável de interesse é o montante do FCL, os resultados se contrapõem à hipótese de Jensen (1986) de que a Governança Corporativa reduziria tal fluxo, com base no fato de que a presença de FCL poderia levar ao desperdício e à ineficiência gerenciais. No entanto, vão ao encontro dos achados de Dylewski (2010), que apontam que empresas com nível de Governança Corporativa mais elevado possuem maiores níveis de caixa do que as demais. Desse modo, uma administração mais comprometida, com melhores práticas de Governança Corporativa, não necessariamente mantém menor nível de liquidez.

Destaca-se, ainda, que, ao contrário do esperado, não foi verificada uma relação significativa e negativa entre o FCL e o Nível 1 de Governança Corporativa. Estes resultados podem ser apenas situacionais ou podem ser explicados pelo fato de que, em alguns ambientes, quando a empresa possui altos níveis de liquidez de caixa, os gestores podem se resguardar, preferindo manter saldo em caixa e não investir, o que resulta em acúmulos de excesso de caixa com o intuito de aumentar os ativos em seu poder. Isto pode levar a subinvestimento corporativo, resultante do não investimento em projetos rentáveis que criem valor para a empresa.

A variável TAM, representada pelo tamanho das empresas, apresentou significância estatística ao nível de 5\%, culminando em uma relação positiva com a variável FCLs. Sob essa ótica, o resultado encontrado corrobora a relação esperada no estudo, estando em conformidade com a teoria de Jensen (1986). Em sinergia, a variável ROE também apresentou significância estatística, sendo validada no modelo estimado nesta pesquisa. Desta forma, pode-se inferir que ela possui uma relação positiva com a variável dependente FCL. Tal fato implica dizer que quanto maior a rentabilidade das empresas, aferida nesse trabalho pela proxy rentabilidade sobre o patrimônio líquido, maior tende a ser o FCL, o que contraria a relação esperada no estudo, conforme balizada na teoria de Jensen (1986). Nessa perspectiva, Wang (2010) evidencia que os FCLs podem proporcionar às empresas oportunidades de investimento que geram mais valores para elas, ocasionando um impacto positivo em seu desempenho.

Por fim, a variável TAXCRESC, que representa a taxa de crescimento das empresas, apresentou significância no modelo e apontou uma relação negativa com a variável FCL. Tal resultado permite inferir que empresas ainda em fase de crescimento, que não atingiram um processo de amadurecimento, tendem a possuir um menor FCL, corroborando as evidências de Jensen e Meckling (1976) e de Jensen (1986), dentro de um contexto de conflito de agência.

\section{CONSIDERAÇÕES FINAIS}

Esta pesquisa objetivou analisar a relação entre os FCLs das organizações e os diferentes níveis de Governança Corporativa presentes no mercado acionário brasileiro. A teoria defendida por Jensen (1986) argumenta que a presença de FCL pode levar ao desperdício e a ineficiências gerenciais e, por isso, os detentores do capital implementam mecanismos de controle para garantir seus interesses, com vistas a minimizar conflitos de interesses presentes na relação de agência.

Os resultados encontrados sugerem que os níveis de governança do mercado acionário brasileiro Novo Mercado e Nível 2 apresentam relação significativa e positiva com o FCL. Isso significa dizer que quanto maiores os níveis de governança praticados pelas organizações, maiores FCLs elas apresentam, quando comparadas às empresas listadas no segmento Tradicional da $[\mathrm{B}]^{3}$. 
Esse resultado refuta a suposição de que a essência da Governança Corporativa é a de minimizar os impactos dos conflitos de agência, mas reforça os resultados apresentados por Dylewski (2010).

No tocante às demais variáveis independentes incluídas no modelo estimado neste estudo, o retorno sobre o patrimônio líquido também apresentou significância estatística e positiva com o FCL, o que implica dizer que quanto maior a rentabilidade das empresas, aferida nesse trabalho pela proxy rentabilidade sobre o patrimônio líquido, maior tende a ser seu FCL, o que contraria a relação esperada no estudo, conforme balizada na teoria de Jensen (1986). Tal fato, também, pode ser algo situacional, assim como pode sugerir um ambiente com boas oportunidades de investimento e, por conseguinte, rentabilidade.

O tamanho da empresa apresentou relação significativa e positiva com o FCL. Já a taxa de crescimento apresentou relação significativa e negativa com o FCL. Sob essa ótica, os resultados encontrados corroboram a relação inicialmente esperada neste estudo, estando em conformidade com a teoria de Jensen e Meckling (1976) e Jensen (1986). Assim, quanto maior o tamanho das empresas, maiores seus FCLs. Já as empresas ainda em fase de crescimento, que não atingiram um processo de amadurecimento, tendem a possuir um menor FCL.

Em resumo, os principais achados da pesquisa sugerem que, dentre os atributos corporativos discutidos por Jensen e Meckling (1976), Jensen (1986) e Dylewski (2010), como possíveis determinantes para o nível de FCL, apenas não parece influenciá-lo o nível de Governança Corporativa 1, ressalvando-se as limitações do estudo, em particular deficiências das definições operacionais das variáveis e um possível viés de seleção introduzido pelos critérios de amostragem. E os demais atributos - Governança Corporativa Nível 2 e Novo Mercado, tamanho, retorno sobre o investimento e taxa de crescimento - são fatores explicativos para o nível de FCL.

Estes resultados contribuem na medida que elucidam, num ambiente sujeito a imperfeições, como os agentes estão agindo e como está se dando essa relação de agência, fornecendo subsídios para a melhor compreensão de como os mecanismos de Governança Corporativa relacionam-se com a teoria dos FCLs. Ademais, fomenta o debate para a realização de novas pesquisas no meio acadêmico, propiciando ampliar a discussão aos diversos stakeholders, dado, inclusive, que a não confirmação da relação esperada traz uma indagação acerca do avanço deste estudo em relação ao cabedal de conhecimento na área.

Quanto às limitações desta pesquisa, compete salientar que os resultados encontrados se limitam à amostra estudada, devido à característica não probabilística do processo de amostragem. Ressalta-se, assim, que a interpretação dos resultados deve ser pautada nesses pontos limitantes, com o intuito de que não haja uma generalização para toda a população, mas que seja um direcionador crítico no processo decisório.

Sugere-se, com vistas a consolidar a teoria dos FCLs, que pesquisas futuras incluam outras variáveis não consideradas neste estudo e que possuam potencial de fundamentar a citada teoria, o que permitirá a continuidade desta investigação. Adicionalmente, outras variáveis exógenas (preços de ações, volume de negociações e variáveis macroeconômicas, por exemplo) poderiam ser empregadas no estudo.

\section{REFERÊNCIAS}

Arruda, G. S., Madruga, S. R., \& Freitas Junior, N. I. (2008). A governança corporativa e a teoria da agência em consonância com a controladoria. Revista de Administração da UFSM, 1(1), 7184.

Avelar, E. A., Cunha, N. G., Boina, T. M., \& Souza, A. A. (2015). Teoria dos Fluxos de Caixa Livres: uma Análise no Mercado Acionário Brasileiro. Anais Congresso USP de Controladoria e Contabilidade, São Paulo, SP, 15. 
Baltagi, B. H. (2005). Econometric analysis of panel data (3a ed.). New York: John Wiley \& Sons.

Brasil, Bolsa, Balcão, ([B] $]^{3}$ (2019). Segmentos de listagem. Recuperado em 28 novembro, 2019, de http://www.b3.com.br/pt_br/produtos-e-servicos/solucoes-para-emissores/segmentos-delistagem/bovespa-mais/

Chi, J. D., \& Lee, D. S. (2010). The conditional nature of the value of corporate governance. Journal of Banking and Finance, 34(2), 350-361.

Dittmar, A., \& Mahrt-Smith, J. (2007). Corporate governance and the value of cash holdings. Journal of financial economics, 83(3), 599-634.

Dylewski, C. (2010). Determinantes do nível de caixa das empresas: análise de amostra de países da América Latina. Dissertação (Mestrado Profissional em Finanças e Economia), Fundação Getúlio Vargas, São Paulo, Brasil.

Fávero, L. P. L., Belfiore, P., Silva, F. L., \& Chan, B. L. (2009). Análise de Dados: Modelagem Multivariada para Tomada de Decisões. Rio de Janeiro: Elsevier.

Ferreira, C. O., Ferreira, P. O., Lamounier, W. M., \& Avelar, E. A. (2021). Fluxos de caixa livres e endividamento em operadoras de planos de saúde. ForScience, 9(1), e00865.

Ferreira, M. A., \& Vilela, A. S. (2004). Why do firms hold cash? Evidence from EMU countries. European Financial Management, 10(2), 295-319.

Francis, B., Hasan, I., Song, L., \& Waisman, M. (2013). Corporate governance and investmentcash flow sensitivity: Evidence from emerging markets. Emerging Markets Review, 15, 57-71. http://doi.org/10.1016/j.ememar.2012.08.002

Gelbecke, E. R., Santos, A., Iudícibus, S., \& Martins, E. (2018). Manual de contabilidade societária: aplicável a todas as sociedades de acordo com as normas internacionais e do CPC. (3a ed.). São Paulo: Atlas.

Gujarati, D. N., \& Porter, D. C. (2011) Econometria Básica (5a ed.). Porto Alegre: AMGH Editora Ltda.

Gujarati, D.N. (2006). Econometria Básica. Rio de Janeiro: Elsevier.

Gupta, G., Mahakud, J., \& Verma, V. (2021), CEO's education and investment-cash flow sensitivity: an empirical investigation. International Journal of Managerial Finance, 17(4), 589-618. https://doi.org/10.1108/IJMF-01-2020-0020

Hedlund, P. R., Zaluski, F. C., Siqueira, C. S., Silva, P. R., Brizolla, M. M. B., Gomes, C. M., \& Mueller, A. A. (2021). A Relação entre Governança Corporativa e Teoria da Agência no Gerenciamento de Conflitos. Gestão e Sociedade, 15(41), 4144-4162.

Huu Nguyen, A., Thuy Doan, D., \& Ha Nguyen, L. (2020). Corporate governance and agency cost: Empirical evidence from Vietnam. Journal of Risk and Financial Management, 13(5), 103. 
Instituto Brasileiro de Governança Corporativa - IBGC (2019). Governança Corporativa. Recuperado de https://www.ibgc.org.br/conhecimento/governanca-corporativa.

Jensen, M. C. (1986). Agency Costs of Free Cash Flow, Corporate Finance, and Takeovers. American Economic Review, 76(2), 323-329.

Jensen, M. C., \& Meckling, W. H. (1976). Theory of the firm: managerial behavior, agency cost and ownership, structure. Journal of Financial Economics, 3, 305-360.

Johnston, J., \& DiNardo, J. (2001). Métodos econométricos (4a Ed.). Portugal: Mcgraw-Hill.

Keynes, J. M. (1936). The general theory of employment, interest, and money. Springer.

Klann, R. C., Gomes, E. C. O., Greuel, M. A., \& Bezerra, F. A. (2014). Influência do Risco Moral e da Accountability nas tomadas de decisões. Contabilidade Vista \& Revista, 25(1), 99-118.

Lambright, K. T. (2009). Agency theory and beyond: Contracted providers' motivations to properly use service monitoring tools. Journal of Public Administration Research and Theory, 19, 207-227.

Lang, L. H. P., Stulz, R. M., \& Walkling, R. A. (1991). A test of the free cash flow hypothesis. Journal of Financial Economics, 29, 315-335.

Lerner, A. F. (2019). Relação do fluxo de caixa livre com o desempenho econômico e os custos de agência. Dissertação (Mestrado em Ciencias Econômicas), UFRGS, Porto Alegre, RS, Brasil.

Lerner, A. F., \& Victor, F. G. (2020). Análise da Influência do Fluxo de Caixa Livre nos Indicadores de Desempenho Econômico em Companhias Abertas Brasileiras. Navus: Revista de Gestão e Tecnologia, 10(1), 1-23.

Li, M., \& Song, L. (2018). Corporate governance, accounting information environment and investment-cash flow sensitivity. International Journal of Accounting \& Information Management, 26(4), 492-507. https://doi.org/10.1108/IJAIM-04-2017-0049

Lodi, J. B. (2000). Governança Corporativa. Rio de Janeiro: Campus.

Machado, J. H., \& Galdi, F. C. (2021). Ativos Intangíveis e Estrutura de Propriedade: Evidências no Mercado Brasileiro. Anais do USP International Conference in Accounting, São Paulo, SP, Brasil, 21.

Macho-Stadler, I., \& Pérez-Castrillo, J. D. (2001). An introduction to the economics of information: incentives and contracts. Oxford University Press on Demand.

Martin, N. C., Santos, L. R., \& Dias Filho, J. M. (2004). Governança Empresarial, Riscos e Controles Internos: A Emergência de um Novo Modelo de Controladoria. Revista Contabilidade \& Finanças, 34(1), 7-22. 
McKnight, P. J., \& Weir, C. (2009). Agency costs, corporate governance mechanisms and ownership structure in large UK publicly quoted companies: A panel data analysis. The Quarterly Review of Economics and Finance, 49(2),139-58.

Modigliani, F., \& Miller, M. H. (1958). The cost of capital, corporation finance and the theory of investment. The American, $1,3$.

Oliveira, F. B., \& Fontes Filho, J. R. (2021). Mudanças nas configurações de governança corporativa e relações de agência: uma análise longitudinal em empresa de capital fechado. Cadernos EBAPE, 19, 510-523.

Raupp, F. M., \& Beuren, I. M. (2008). Metodologia da pesquisa aplicável às ciências sociais. In I. M. Beuren (Org.). Como elaborar trabalhos monográficos em Contabilidade: teoria e prática. São Paulo: Atlas.

Sehrawat, N. K., Kumar, A., Lohia, N., Bansal, S., \& Agarwal, T. (2019). Impact of Corporate Governance on Earnings Management: Large Sample Evidence from India. Asian Economic and Financial Review, 9(12), 1335.

Shleifer, A., \& Vishny, R.W. (1997). A Survey of Corporate Governance. The Journal of Finance. $\begin{array}{lllll}\text { Recuperado em } & 27 & \text { novembro, } & 2019, & \text { de }\end{array}$ https://scholar.harvard.edu/files/shleifer/files/surveycorpgov.pdf

Silva, B. A. O., Caixe, D. F., \& Krauter, E. (2019). Governança Corporativa e Sensibilidade Investimento-Fluxo de Caixa no Brasil. Revista Brasileira de Finanças, 17(2), 72-86.

Sprenger, C., \& Lazareva, O. (2021). Corporate governance and investment-cash flow sensitivity: Evidence from Russian unlisted firms. Journal of Comparative Economics, 49(3), september. https://doi.org/10.1016/j.jce.2021.05.004

Waly, N. A., Sasongko, N., \& Achyani, F. (2021). Effect of Free Cash Flow, Enterprise Risk Management Disclosure and Sustainability Report on Company Value With Corporate Governance as Moderating Variable. SENTRALISASI, 10(2), 140-146.

Wang, G. Y. (2010). The impacts of free cash flows and agency costs on firm performance. Journal of Service Science and Management, 3(Dec), 408-418.

Yeo, H. J. (2018). Role of free cash flows in making investment and dividend decisions: The case of the shipping industry. The Asian Journal of Shipping and Logistics, 34(2), 113-118.

Zhang, X. (2020). Corporate Governance and Agency Costs of Free Cash Flow in Acquisitions (Doctoral dissertation), Concordia University. 